\title{
Characterization of IPS-LCD by Reduced One-dimensional Model
}

\author{
Byung-Hee Park and Yeon-Ho Lee* \\ Department of Information and Communication Engineering, Sungkyunkwan University, \\ Suwon 440-746, Korea
}

(Received March 14, 2011 : revised June 1, 2011 : accepted June 3, 2011)

\begin{abstract}
Based on the distinctive characteristics observed in the intensity transmittance of an IPS-LC panel, the previous one-dimensional model is greatly reduced such that only a few data points and their interpolations predict the intensity transmittance of an IPS-LCD with a small error for arbitrary gray levels. Experimental procedure and numerical methods are described in detail.
\end{abstract}

Keywords: In-plane switching, Liquid crystal display, One-dimensional model, Twist angle

OCIS codes : (120.2040) Displays; (160.3710) Liquid crystals; (230.3720) Liquid-crystal devices

\section{INTRODUCTION}

In-plane switching(IPS) technology imparts to Liquid Crystal Displays(LCDs) many desirable properties such as high contrast ratio, no gamma shift, stable color reproduction, wide viewing angle, and so on. These advantages make in-plane switching technology a strong candidate for high definition (HD) TVs, computer monitors and mobile appliances[1].

Inter-digitated electrodes fabricated on the same substrate are a characteristic structure of IPS-LC Panel, and produce a strong vertical electric field as well as the horizontal one. The coexistence of the vertical and horizontal fields generates a complicated spatial distribution of LC molecules. In this case, only the result of numerical methods conducted by including Berreman's Q tensor representation can describe the multidimensional director configurations $[2,3]$. Once the distribution of directors is given, the intensity transmittance can be obtained by various methods : finite-difference timedomain method[4, 5], beam propagation method[6], reducedorder grating method or a combined method [7-9]. Recently H. J. Cho, et. al. have proposed a simple one-dimensional model for the director distribution in an in-plane switching liquid crystal panel and have calculated the intensity transmittance by using the Jones matrix method [10]. They have reported an excellent agreement between experimental data and computer simulations.

In this paper we report an optical effect recurring in the experimental data of the intensity transmittance through an IPS-LC panel and propose a greatly reduced experimental procedure for characterizing an IPS-LC panel. The new method allows us to save time both in the experiment and computer simulation.

\section{EXPERIMENT}

We first measure the light intensities transmitted through an IPS-LC panel by changing the polarization of the input beam and by changing the signal voltage applied to an LC panel. We collect data following the general procedure for the transmitted light intensity[10, 11]. FIG. 1 shows a schematic diagram for the experimental setup. In the experiment, we use a commercial LCD manufactured by LG Display for mobile displays. A beam from a He-Ne laser is first expanded by a beam expander to a diameter of $30 \mathrm{~mm}$ and then given circular polarization by a quarter-wave plate. The beam is given linear polarization by an input polarizer before it is incident to the IPS-LC panel. To monitor a possible fluctuation in the input beam, we measure the beam intensity with photo detector PD1. We measure the polarization state of the modulated beam from the LCD with an output polarizer and photo detector PD2. The intensity transmittance is defined by the ratio between the output intensity measured at PD2 to the input intensity measured at PD1. To cancel out the system noise, we normalize the intensity transmittance by the loss factor squared. The loss factor will be discussed later.

Our IPS-LC panel can display 64 gray levels. When we

\footnotetext{
*Corresponding author: yeonlee@ece.skku.ac.kr
} 
input a two dimensional image of a gray level to the LCD driver, it is converted to a driving voltage and is applied to the LCD panel. We measure the transmitted intensity by changing the input gray level, and the polarization states of the input and output laser beams. For a fixed gray level, we measure the loss factor squared by measuring the maximum and the minimum output intensities for possible combinations of the input polarizer and the output polarizer rotations, and dividing their sum by the input beam intensity.

FIG. 2 shows the experimental data of the transmitted intensities for 4 gray levels, 0, 16, 48 and 63. For an

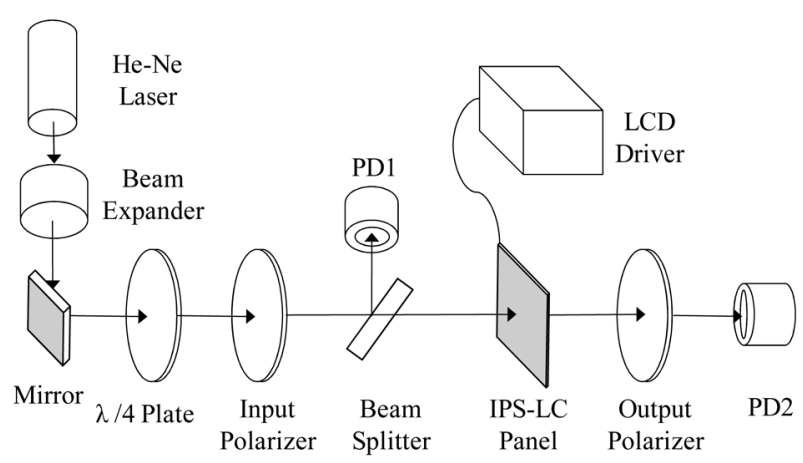

FIG. 1. Experimental schematic for intensity transmittance through an IPS-LCD. IPS-LCD, In-Plane Switching Liquid Crystal Display; PD, Photo Detector.

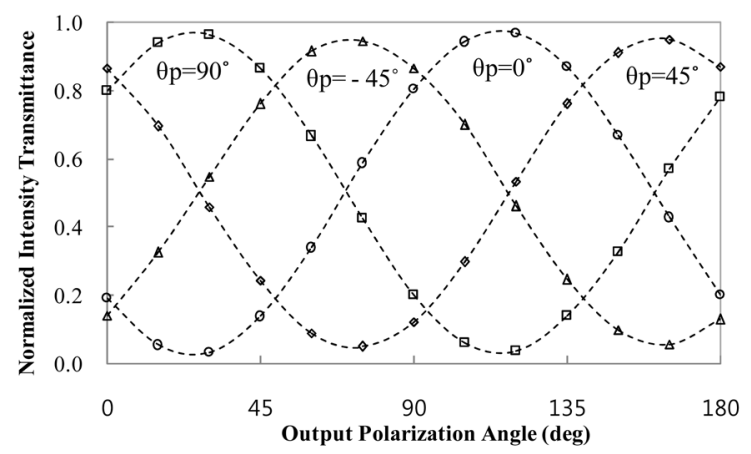

(a) Gray 0

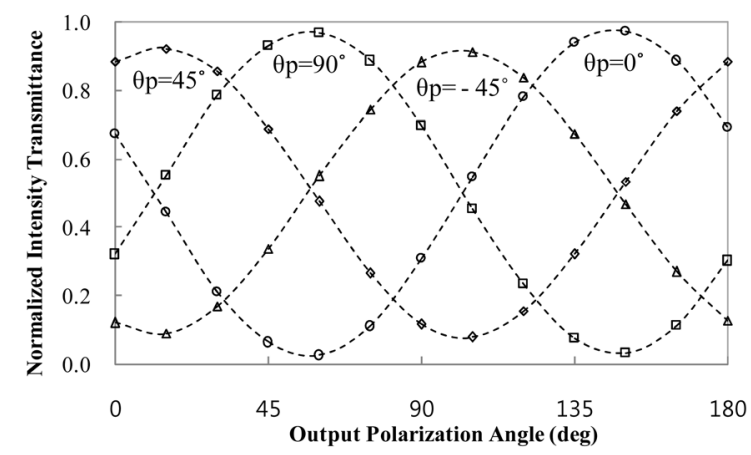

(c) Gray 48 input polarization direction, $\theta_{p}$, fixed at one of $-45,0,45$ and 90 degrees, the measured intensity transmittance is first normalized by the loss factor squared, and plotted as a function of the output polarization direction. In the figure, the experimental data are shown by circles. The curves are used to connect the data for a better visibility. We notice from the figure that the transmittance curve appears the same whenever the input polarization is changed by 90 degrees, although two curves are displaced to each other. For example, when the gray level is 63 , the transmittance curves of $\theta_{p}=0^{\circ}$ and $\theta_{p}=90^{\circ}$ look identical except for a shift in position. The same is true for the transmittance curves of $\theta_{p}=45^{\circ}$ and $\theta_{p}=-45^{\circ}$. When we check with the experimental data, two transmittance curves are identical with a root-mean-square error less than 0.15 .

\section{ONE-DIMENSIONAL MODEL}

When there is no voltage applied to IPS-LCD, LC molecules are parallel to each other, and align with the rubbing direction of two substrates. The rubbing direction makes an angle of 7 degrees in our IPS-LCD with respect to the $+y$-axis. FIG. 3(a) is a 3-D perspective of LC molecules with no applied voltage and FIG. 3(b) is its side view. When a voltage is applied to LCD, LC molecules start to rotate toward the $+x$-axis, whose angle is called the twist

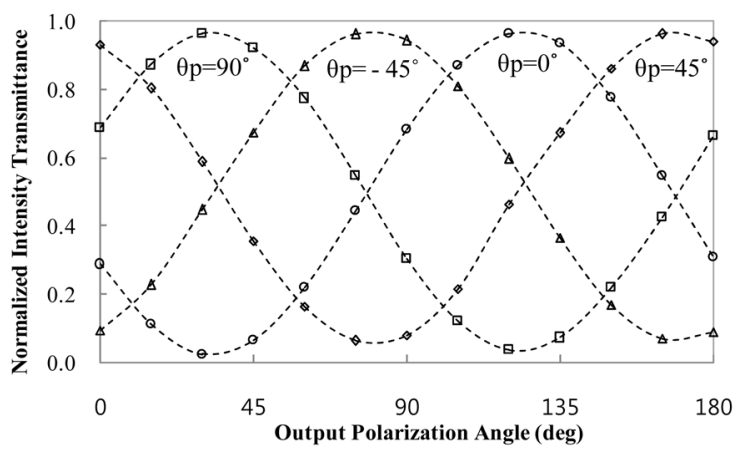

(b) Gray 16

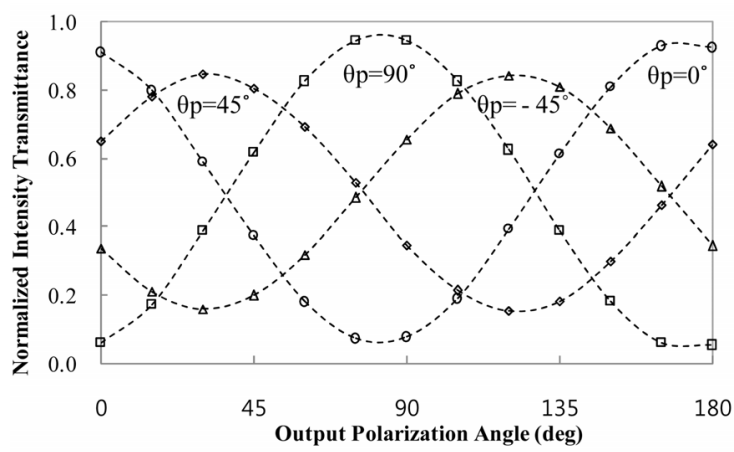

(d) Gray 63

FIG. 2. Normalized intensity transmittance is measured as a function of output polarization direction. $\theta_{p}$ is the input polarization direction. Diamonds, squares, circles and triangles represent experimental data. Dotted curves are interpolations for visibility. (a) Gray 0 (b) Gray 16 (c) Gray 48 (d) Gray 63. 


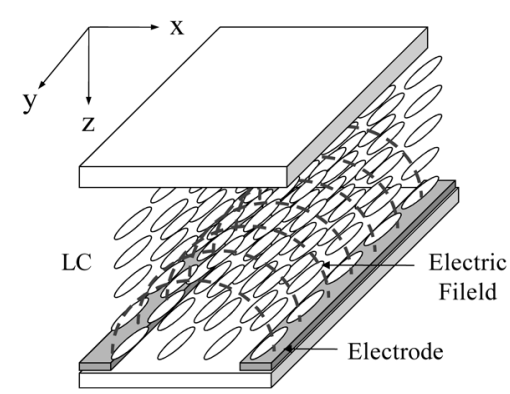

(a)

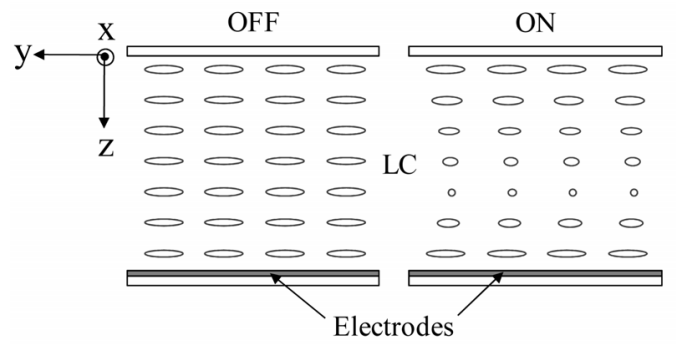

(b)

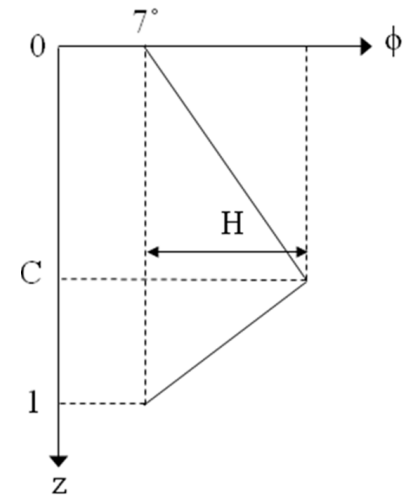

(c)

FIG. 3. (a) Schematics of LC molecules in IPS-LCD with no applied voltage. (b) Side views of IPS-LCD with a voltage applied. (c) One-dimensional model for twist angle of liquid crystal molecules as described in equations $1 \mathrm{a}$ and $1 \mathrm{~b}$.

angle. Although the electric field intensity is the maximum at the bottom substrate on which the electrodes are built, the maximum twist of LC molecule occurs near the center region of the LCD, because $\mathrm{LC}$ molecules are not free to rotate near the top and bottom substrates. The distribution of LC molecules is so complicated in IPS-LCD that only a 3-D model may predict the exact distribution of LC molecules. However, in this paper, we use a simple 1-D model of IPS-LCD in which it is assumed that the twist angle of LC molecules increases linearly from the top substrate to the center region, and then decreases to the bottom substrate. In this model, the peak twist angle $\mathrm{H}$ and the location of the peak $\mathrm{C}$ are two parameters that are supposed to be determined from the experimental data. FIG. 3(c) shows our 1-D model of IPS-LCD used in the simulations. An analytic expression for the twist angle of LC molecule is given by

$$
\begin{aligned}
& \phi=\frac{H}{C} z+7^{\circ}, \quad(\mathrm{z} \leq \mathrm{C}) \\
& \phi=-\frac{H}{1-C}(z-C)+\left(7^{\circ}+H\right),(\mathrm{z} \geq \mathrm{C})
\end{aligned}
$$

If the system parameters $\mathrm{C}$ and $\mathrm{H}$ are given, we can calculate the intensity transmitted through LCD by using the standard procedure involving Jones Matrix. In this case, the beam monitored by PD1 gives the input polarization state, while that monitored by PD2 gives the output polarization state. The two polarization states are related by the Jones Matrix equation as

$$
\left[\begin{array}{c}
E_{x}^{\text {out }} \\
E_{y}^{\text {out }}
\end{array}\right]=J\left(\theta_{P}\right) J_{I P S-L C}\left[\begin{array}{c}
E_{x}^{\text {in }} \\
E_{y}^{\text {in }}
\end{array}\right],
$$

where $J\left(\theta_{p}\right)$ is the Jones Matrix of the output polarizer with a rotation angle $\theta_{p}$, and $J_{I P S-L C}$ is that of IPS-LC panel. In order to find $J_{I P S-L C}$, we slice IPS-LC panel into 100 hypothetical layers, starting from the top to bottom substrate. In a layer, LC molecules are assumed parallel to each other, with a fixed twist angle, so that we may treat the layer as a waveplate of a fixed birefringence. The Jones matrix of the k-th slice is given by,

$$
J_{k}=\left[\begin{array}{cc}
\cos \phi_{k} & \sin \phi_{k} \\
-\sin \phi_{k} & \cos \phi_{k}
\end{array}\right]\left[\begin{array}{cc}
e^{-i \beta_{k}} & 0 \\
0 & e^{i \beta_{k}}
\end{array}\right]\left[\begin{array}{cc}
\cos \left(-\phi_{k}\right) & \sin \left(-\phi_{k}\right) \\
-\sin \left(-\phi_{k}\right) & \cos \left(-\phi_{k}\right)
\end{array}\right],
$$

where $\theta_{k}$ is the twist angle of LC molecules in the k-th slice, which is measured from the $+\mathrm{y}$-axis toward the $\mathrm{x}$-axis. The phase retardation of the k-th slice, $\beta_{k}$, is defined by $\beta_{k}=\left(n_{e}-n_{o}\right)(\pi d / 100 \lambda)$, where the extraordinary refractive index $n_{e}=1.5854$, ordinary refractive index $n_{o}=1.4825$, the wavelength $\lambda=632.8 \times 10^{-9} \mathrm{~m}$, the thickness of the IPS-LC panel $\mathrm{d}=3.2 \times 10^{-6} \mathrm{~m}$, and the number 100 signifies that the LC panel is sliced into 100 layers. The third matrix on the right side of Eq. (3) is for transforming the polarization state of the input laser beam into the coordinates of the ordinary and extraordinary axes of the LC molecule. The first matrix is for transforming the polarization state of the output beam into the laboratory coordinates. Then, the Jones matrix of IPS-LC panel can be obtained from $J_{I P S-L C}=J_{100} J_{99} \ldots J_{2} J_{1}$. The Jones matrix of the input polarizer or output polarizer is obtained by the same method as

$$
J(\theta)=\left[\begin{array}{cc}
\cos \theta & \sin \theta \\
-\sin \theta & \cos \theta
\end{array}\right]\left[\begin{array}{ll}
1 & 0 \\
0 & 0
\end{array}\right]\left[\begin{array}{cc}
\cos \theta & -\sin \theta \\
\sin \theta & \cos \theta
\end{array}\right]=\left[\begin{array}{cc}
\cos ^{2} \theta & -\cos \theta \sin \theta \\
-\cos \theta \sin \theta & \sin ^{2} \theta
\end{array}\right],
$$

where $\theta$ is the rotation angle of the input polarizer or output polarizer, measured from the $\mathrm{y}$-axis toward the $\mathrm{x}$-axis.

The intensity transmittance $\mathrm{T}$ is given by the ratio of the measured output intensity $\mid \vec{E}^{\text {in }\left.\right|^{2}}$ to $\left(\left|\vec{E}^{\text {out }}\right|_{\text {max }}^{2}+\left|\vec{E}^{\text {out }}\right|_{\text {min }}^{2}\right)$ 


$$
T=\frac{\left|\vec{E}^{\text {out }}\right|^{2}}{\left(\left|\vec{E}^{\text {out }}\right|_{\text {max }}^{2}+\left|\vec{E}^{\text {out }}\right|_{\text {min }}^{2}\right)}=\frac{\left|\vec{E}^{\text {out }}\right|^{2}}{\left|\vec{E}^{\text {in }}\right|^{2} c^{2}},
$$

where $\left|\vec{E}^{\text {out }}\right|_{\max }^{2}$ and $\left|\vec{E}^{\text {out }}\right|_{\min }^{2}$ are the maximum and minimum output intensities that are measured by fixing the input beam polarization state. It is customary to write $\left(\left|\vec{E}^{\text {out }}\right|_{\max }^{2}+\left|\vec{E}^{\text {out }}\right|_{\text {min }}^{2}\right)$ as $\left|\vec{E}^{\text {in }}\right|^{2} c^{2}$, where c is called the loss factor[11, 12]. It is noted that $1-c^{2}$ represents the system loss for a unit input intensity.

\section{NUMERICAL METHOD}

When a gray level is fixed, we obtain the twist angle of LC molecules by fitting Eq. (1) to the experimental data of intensity transmittance. In this case, one might think that more experimental data would result in more accurate values of $\mathrm{C}$ and $\mathrm{H}$. However, as we showed in FIG. 2, the intensity transmittance curves repeat themselves every 90 degrees of polarization rotation. In other words, only experimental data with polarization angles in a range of 90 degrees can contribute to the curve fitting. Therefore, we use only two polarization angles for a fixed gray level, which are 45 and 90 degrees. In FIG. 4, the bold lines represent fitting of Eq. (1) to the experimental data of intensity transmittance for the polarization angle of 45 and 90 degrees, when the gray level is fixed to 16 . In this case, $\mathrm{C}$ and $\mathrm{H}$ are calculated to be 0.88 and -17.8 degrees, respectively, as can be seen from FIG. 5. We repeat the curve fitting for gray levels of $0,16,48$ and 63, and interpolate $\mathrm{C}$ and $\mathrm{H}$ to obtain smooth curves as shown in FIG. 5. Even if we use intensity transmittances of only two polarization rotations and four gray levels, the interpolations of $\mathrm{C}$ and $\mathrm{H}$ predict intensity transmittances with very small errors, compared with the experimental data.

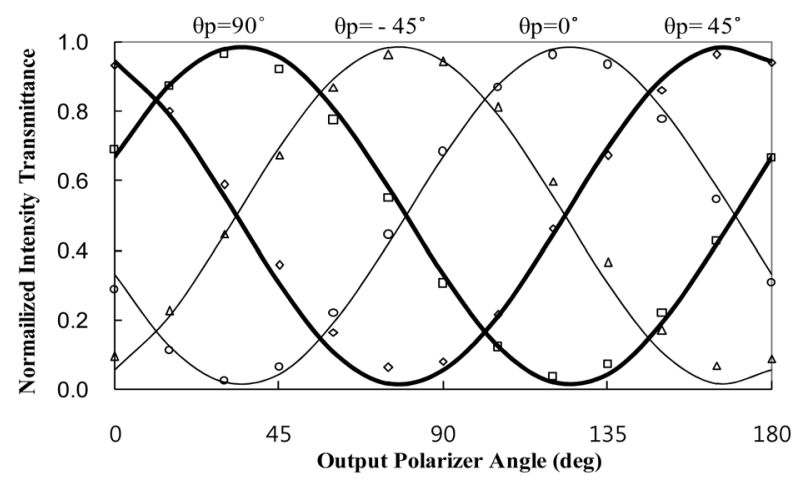

FIG. 4. Triangles $\left(\theta_{p}=-45^{\circ}\right), \operatorname{circles}\left(\theta_{p}=0^{\circ}\right)$, diamonds $\left(\theta_{p}=45^{\circ}\right)$, and squares $\left(\theta_{p}=90^{\circ}\right)$ represent experimental data of intensity transmittance for gray level 16 . Thick curves are fittings of one-dimensional model to the data. Thin curves are predictions of the one-dimensional model with the obtained values of $\mathrm{C}$ and $\mathrm{H}$.
In FIG. 5, we know the values of $\mathrm{C}$ and $\mathrm{H}$ only for gray levels of $0,16,48$ and 63 . For gray level of 32, for example, $\mathrm{C}$ and $\mathrm{H}$ are obtained from polynomial interpolation given as follows,

$$
\begin{aligned}
& C(x)=C\left(x_{1}\right) L_{1}(x)+C\left(x_{2}\right) L_{2}(x)+C\left(x_{3}\right) L_{3}(x)+C\left(x_{4}\right) L_{4}(x), \\
& H(x)=H\left(x_{1}\right) L_{1}(x)+H\left(x_{2}\right) L_{2}(x)+H\left(x_{3}\right) L_{3}(x)+H\left(x_{4}\right) L_{4}(x),
\end{aligned}
$$

where

$$
\begin{aligned}
& L_{1}(x)=\frac{\left(x-x_{2}\right)\left(x-x_{3}\right)\left(x-x_{4}\right)}{\left(x_{1}-x_{2}\right)\left(x_{1}-x_{3}\right)\left(x_{1}-x_{4}\right)}, \\
& L_{2}(x)=\frac{\left(x-x_{1}\right)\left(x-x_{3}\right)\left(x-x_{4}\right)}{\left(x_{2}-x_{1}\right)\left(x_{2}-x_{3}\right)\left(x_{2}-x_{4}\right)}, \\
& L_{3}(x)=\frac{\left(x-x_{1}\right)\left(x-x_{2}\right)\left(x-x_{4}\right)}{\left(x_{3}-x_{1}\right)\left(x_{3}-x_{2}\right)\left(x_{3}-x_{4}\right)}, \\
& L_{4}(x)=\frac{\left(x-x_{1}\right)\left(x-x_{2}\right)\left(x-x_{3}\right)}{\left(x_{4}-x_{1}\right)\left(x_{4}-x_{2}\right)\left(x_{4}-x_{3}\right)},
\end{aligned}
$$

In the above equations, $x$ is a gray level, and $x_{1}=0$, $x_{2}=16, x_{3}=48$ and $x_{4}=63$. With $\mathrm{C}$ and $\mathrm{H}$ obtained by interpolation, we calculate the intensity transmittances for gray level 32 for four input polarization angles. In FIG. 6, curves represent the calculated intensity transmittances, and dots the experimental data.

To check the validity of our model, we estimate the

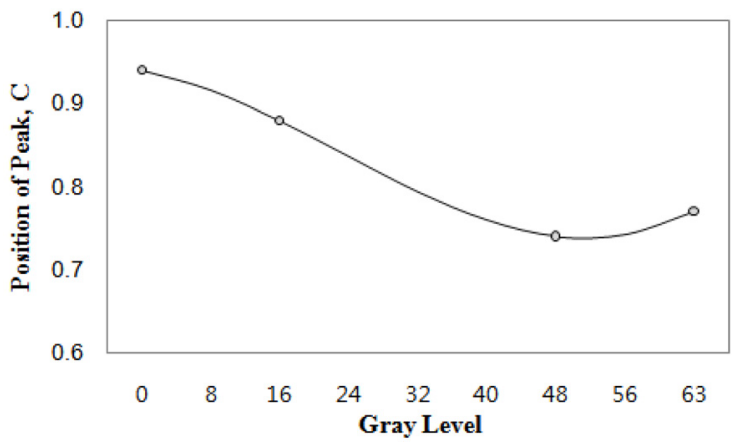

(a) Parameter $\mathrm{C}$

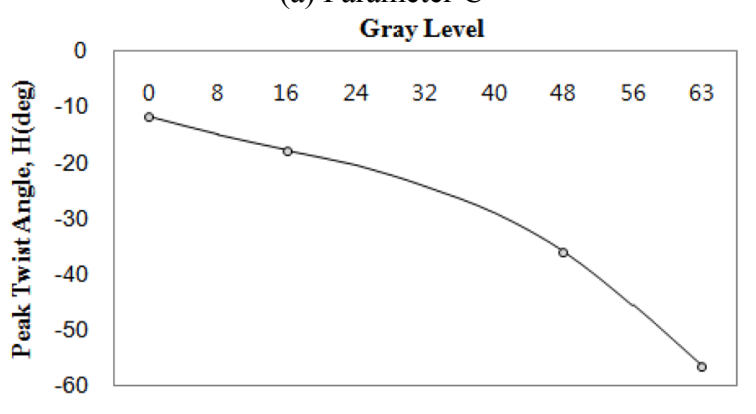

(b) Parameter $\mathrm{H}$

FIG. 5. Circles are $\mathrm{C}$ and $\mathrm{H}$ obtained from fitting of Eq. (1) to experimental data. Curves are polynomial interpolations for (a) parameter $\mathrm{C}$, and (b) parameter $\mathrm{H}$. 


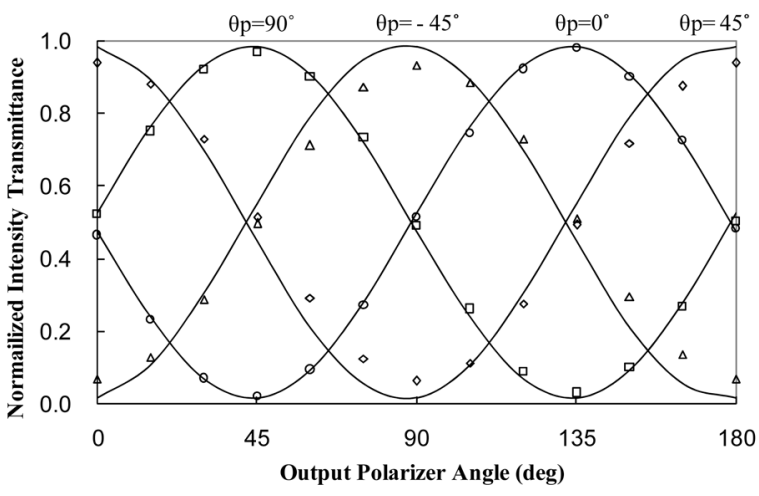

FIG. 6. Triangles $\left(\theta_{p}=-45^{\circ}\right), \operatorname{circles}\left(\theta_{p}=0^{\circ}\right)$, diamonds $\left(\theta_{p}=45^{\circ}\right)$, and squares $\left(\theta_{p}=90^{\circ}\right)$ represent the experimental data for gray 32. The curves represent the predictions of our model with the values of $\mathrm{C}$ and $\mathrm{H}$ obtained from interpolations.

TABLE 1. Errors of $\mathrm{C}$ and $\mathrm{H}$ between our model and the previous model. Bold face denotes the maximum errors

\begin{tabular}{c|c|c}
\hline \hline Gray & Error of C & Error of H \\
\hline 0 & $2.2 \%$ & $3.5 \%$ \\
\hline 8 & $1.9 \%$ & $4.9 \%$ \\
\hline 16 & $\mathbf{3 . 5 \%}$ & $2.0 \%$ \\
\hline 24 & $3.3 \%$ & $-5.2 \%$ \\
\hline 32 & $0.5 \%$ & $-\mathbf{6 . 6 \%}$ \\
\hline 40 & $-2.6 \%$ & $-3.6 \%$ \\
\hline 48 & $-2.6 \%$ & $-0.5 \%$ \\
\hline 56 & $-0.9 \%$ & $4.8 \%$ \\
\hline 63 & $-1.3 \%$ & $-0.3 \%$ \\
\hline
\end{tabular}

error in $\mathrm{C}$ and $\mathrm{H}$ in Table 1. Our model produces $\mathrm{C}$ and $\mathrm{H}$ as shown in FIG. 5, which are obtained by the experimental data of intensity transmittances for two polarization rotations, 45 and 90 degrees, and four gray levels, 0, 16, 48 and 63 . We have conducted further experiments to obtain intensity transmittances for 9 polarization rotations $(-90,-60,-45,-30,0,30,45,60$, and 90 degrees), and 9 gray levels $(0,8,16,24,32,40,48,56$, and 63$)$. We have repeated curve fittings to these experimental data to obtain, so called, " $\mathrm{C}$ and $\mathrm{H}$ of all data". Table 1 shows percent errors between $\mathrm{C}$ and $\mathrm{H}$ of our model and those of all data. We note that the maximum error of $\mathrm{C}$ is estimated to be $3.5 \%$ and the maximum error of $\mathrm{H}$ to be $6.6 \%$. It shows that our model produced little error.

Next, we compare the intensity transmittances of our model and the experimental data. The second column of Table 2 shows percent errors between intensity transmittances predicted by our model and those measured in the experiment. The third column shows percent errors between intensity transmittances predicted with $\mathrm{C}$ and $\mathrm{H}$ that are obtained from all data, and the experimental data. The fourth column
TABLE 2. The second column is the errors between the experimental data of intensity transmittance and the predictions of our model. The third column is the errors between the experimental data and the predictions of the previous model. The last column is the difference between two errors. Our model uses the experimental data of only two polarization rotations and four gray levels. The previous model uses every possible rotations of the input polarizer and values of the gray level

\begin{tabular}{c|c|c|c}
\hline \hline Gray & Our model & Previous model & Difference \\
\hline 0 & $6.1 \%$ & $6.3 \%$ & $-0.2 \%$ \\
\hline 8 & $6.8 \%$ & $6.8 \%$ & $0.0 \%$ \\
\hline 16 & $7.1 \%$ & $7.1 \%$ & $0.0 \%$ \\
\hline 24 & $8.8 \%$ & $7.1 \%$ & $1.7 \%$ \\
\hline 32 & $8.3 \%$ & $7.2 \%$ & $1.1 \%$ \\
\hline 40 & $7.5 \%$ & $7.8 \%$ & $-0.3 \%$ \\
\hline 48 & $7.9 \%$ & $7.7 \%$ & $0.2 \%$ \\
\hline 56 & $11.8 \%$ & $8.6 \%$ & $3.2 \%$ \\
\hline 63 & $10.9 \%$ & $10.6 \%$ & $0.4 \%$ \\
\hline
\end{tabular}

TABLE 3. Values of $\mathrm{H}$ in our IPS-LC panel. At the highest gray level of $63, \mathrm{H}$ is much less than $90^{\circ}$, showing that our IPS-LC panel operates in the linear region

\begin{tabular}{c|c}
\hline \hline Gray & $\mathrm{H}$ (degree) \\
\hline 0 & 11.746 \\
\hline 8 & 15.025 \\
\hline 16 & 17.762 \\
\hline 24 & 20.590 \\
\hline 32 & 24.146 \\
\hline 40 & 29.064 \\
\hline 48 & 35.982 \\
\hline 56 & 45.533 \\
\hline 63 & 56.551 \\
\hline
\end{tabular}

is the difference between the two percent errors. The comparison shows that our model agrees excellently with the experimental data, with the maximum error less than $12 \%$. When all data are used, the error is reduced only by $3.2 \%$. The values of $\mathrm{H}$ in Table 3 show that our IPS-LC panel operates in the linear region.

\section{CONCLUSIONS}

We have measured the intensity transmittance through an IPS-LC panel by changing the gray levels, and by changing the directions of the input polarizer and the output polarizer. From the experimental data we have observed that the intensity transmittance curve repeats itself, in its 
shape, every 90 degree of the polarization direction. Next, by fitting the one-dimensional model to the experimental data, we have measured the parameters $\mathrm{C}$ and $\mathrm{H}$ as functions of gray levels. The curve fitting shows that the $\mathrm{C}$ and $\mathrm{H}$ curves are more sensitive to the values at the gray levels near zero and near the maximum 63 . Based on these new observations, we reform the one dimensional model such that it uses only the experimental data of polarization rotations of 45 and 90 degrees, and the experimental data of four gray levels of $0,16,48$, and 63 . The previous model uses every possible rotation of the input polarizer and every possible value of the gray levels on the premise that more data would produce more accurate fitted parameters of LC panel.[10] When the predictions of our model, for $\mathrm{C}$ and $\mathrm{H}$, are compared with those of previous model, the error is estimated to be less than 3.5\% and $6.6 \%$ for $\mathrm{C}$ and $\mathrm{H}$, respectively. The predictions of our model are also compared with the experimental data of intensity transmittance. In that case, the error is estimated to be less than $11.8 \%$. Since the previous model produces the maximum error of $10.6 \%$, we can conclude that the errors of $11.8 \%$ and $10.6 \%$ just result from the limit of the one dimensional model. Although our model uses a limited amount of experimental data in determining the parameters $\mathrm{C}$ and $\mathrm{H}$, the predictions for the parameters and the intensity transmittance are excellent when they are compared with the experimental data.

\section{ACKNOWLEDGMENT}

This paper was supported by Faculty Research Fund, Sungkyunkwan University, 2010

\section{REFERENCES}

1. N. Aoki, S. Komura, T. Furuhashi, M. Adachi, O. Itou, T. Miyazawa, and M. Ohkura, "Advanced IPS technology for mobile applications," J. Soc. Info. Display 15/1, 23-29 (2007).

2. D. W. Berreman and S. Meiboom, "Tensor representation of Oseen-Frank strain energy in uniaxial cholesterics," Phys. Rev. A 30, 1955-1959 (1984).

3. P. G. de Gennes and J. Prost, The Physics of Liquid Crystals (Oxford Science, Oxford, UK, 1993).

4. B. Witzigmann, P. Regli, and W. Fichtner, "Rigorous electromagnetic simulation of liquid crystal displays," J. Opt. Soc. Am. A 15, 753-757 (1998).

5. E. E. Kriezis and S. J. Elston, "Finite-difference time domain method for light wave propagation within liquid crystal devices," Opt. Comm. 165, 99-105 (1999).

6. E. E. Kriezis and S. J. Elston, "Wide-angle beam propagation method for liquid-crystal device calculations," Appl. Opt. 39, 5707-5714 (2000).

7. K. Rokushima and J. Yamakita, "Analysis of anisotropic dielectric gratings," J. Opt. Soc. Am. 73, 901-908 (1983).

8. O. A. Peverini, D. Olivero, C. Oldano, D. K. G. de Boer, R. Cortie, R. Orta, and R. Tascone, "Reduced-order model technique for the analsis of anisotropic inhomogeneous media: application to liquid-crystal display," J. Opt. Soc. Am. A 19, 1901-1909 (2002).

9. D. A. Yakovlev, V. I. Tsoy, and V. G. Chigrinov, "Advanced tools for modeling of 2D-optics of LCDs," SID 05 Digest, 58-61 (2005).

10. H. J. Cho, D. H. Kim, and Y. H. Lee, "A simple distribution model of the twist angle in an in-plane switching liquid crystal display," J. Opt. A: Pure Appl. Opt. 11, 105409 (2009).

11. H. Kim and Y. H. Lee, "Unique measurement of the parameters of a twisted-nematic liquid-crystal display," Appl. Opt. 44, 1642-1649 (2005).

12. M. Yamauchi and T. Eiju, "Optimization of twisted-nematic liquid-crystal panels for spatial light phase modulation," Opt. Comm. 115, 19-25 (1995). 